\title{
Assessing prior knowledge of statistics in students attending an online university
}

Vanessa Fernández-Chamorro

Sonia Pamplona

María José Pérez-Fructuoso

Universidad a Distancia de Madrid (UDIMA)

Journal of Computing in Higher Education 32, pages182-202(2020)

https://doi.org/10.1007/s12528-019-09236-9

\begin{abstract}
This study analyzes the relationship between the entry path to a degree and the prior statistical competence of students taking a Statistics and Probability course at an online university. We assessed students' prior knowledge by administering a pretest of the information covered in the course analyzed. The sample includes 108 students from different schools of an online university. According to the statistical analysis, students have certain difficulty understanding some concepts related to Probability and Descriptive Statistics, and the entry path affects the students' understanding of these concepts.
\end{abstract}

